BP-SY-2-3

\title{
Standard radical bile duct surgery focused on vascular reconstruction
}

\author{
Shin HWANG* \\ Department of Surgery, Asan Medical Center, University of Ulsan College of Medicine, Seoul, Korea
}

Lecture: Since the mid bile duct cancers often involve the intrapancreatic bile duct, resection of the extrahepatic bile duct results in a tumor-positive distal resection margin. Extended extrahepatic bile duct resection is a surgical procedure to obtain a tumor-free distal resection margin during extrahepatic bile duct resection, in which R0 resection can be achieved without performing pancreatoduodenectomy. The extent of intrapancreatic bile duct excavation was designed to resemble making a several cm-long funnel. The surgical procedure for pancreatic excavation was derived from those used for choledochal cyst excision. Special attention should be paid to prevent pancreatic leak at the bile duct excavation portion at the pancreatic head. If the proximal extrahepatic bile duct is tumor-positive, major or parenchyma-preserving hepatectomy is necessary to achieve R0 resection. Resection of segment I and segment IV with bile duct resection offers very wide operative field and allows wider extent of hilar bile duct resection, thus presents the most common type of parenchyma-preserving hepatectomy. When the portal vein is focally involved by the tumor, hilar portal vein wedge resection and roofing patch venoplasty using a autograft or homograft vein patch is a useful option to facilitate complete tumor resection in patients undergoing bile duct resection. Regarding roofing patch venoplasty, it is important to make the portal vein wall defect as small as possible as well as to make the size of the vein patch either twice or three times larger than the defect size at the portal vein wall. The patch should be large enough to make the patch roof redundant. Even if the patch roof appears to be noticeably redundant at the time of operation, it is vulnerable to shrinkage due to compression of the jejunal loop and through degenerative changes of the patch per se. The availability of vein autografts or homografts facilitates combined vascular resection and reconstruction during aggressive surgery for hepatobiliary malignancies. 